\section{SAFETY AND EFFICACY OF INTRATUMORAL IPILIMUMAB WITH IV NIVOLUMAB IN METASTATIC MELANOMA. THE NIVIPIT TRIAL}

${ }^{1}$ Lambros Tselikas*, ${ }^{1}$ Caroline Robert, ${ }^{2}$ Stephane Dalle, ${ }^{3}$ Nicolas Meyer, ${ }^{4}$ Celeste Lebbe ${ }^{1}$ Samy Ammari, ${ }^{1}$ François-Xavier Danlos, ${ }^{1}$ Severine Roy, ${ }^{1}$ Camille Jannin, 'Siham Farhane, ${ }^{1}$ Severine Mourad, ${ }^{1}$ Guillaume Escriou, ${ }^{1}$ Thibault Raoult, ${ }^{1}$ Jean-Yves Scoazec, ${ }^{1}$ Matthieuu Texier, ${ }^{1}$ Nathalie Chaput-Gras, 'Laurence Zitvogel, ${ }^{1}$ Thierry De Baere, ${ }^{5}$ Aurélien Marabelle. ' Gustave Roussy, Villejuif, France; ${ }^{2} \mathrm{HCL}$, Claude Bernard University, Lyon, France; ${ }^{3}$ UUCT-O, CHU Toulouse, Toulouse, France; ${ }^{4}$ APHP, Paris, France; ${ }^{5}$ Gustave Roussy, Villejuif, France

Background Intratumoral (IT) administration of Ipilimumab (Ipi), could maximize its dose/efficacy ratio while preventing its on-target/off-tumor systemic adverse events. We report the results of a randomized multicenter Phase $1 \mathrm{~b}$ study comparing IT vs IV Ipi with intravenous nivolumab (nivo) in patients with metastatic melanoma.

Methods Previously untreated metastatic melanoma patients were randomly assigned $1: 2$, to receive IV Nivo $(1 \mathrm{mg} / \mathrm{kg})$ in combination with either IV Ipi $(3 \mathrm{mg} / \mathrm{kg})$ or $10 \mathrm{x}$ lower dose IT Ipi $(0.3 \mathrm{mg} / \mathrm{kg}) \mathrm{Q} 3 \mathrm{~W}$ for 4 doses, followed by Nivo $3 \mathrm{mg} /$ $\mathrm{kg}$ Q2W for up to 2 years. The primary objective was to compare $\geq$ grade 3 irAE rates at 6 months. Secondary objectives were to assess anti-tumor efficacy and related predictive immune biomarkers.Fresh tumor biopsies pre $\&$ on-treatment on both injected and non-injected tumors were analyzed by flow-cytometry, and soluble factors from their supernatant were titrated with Meso-Scale-Discovery ${ }^{\circledR}$ multiplex. Fresh sequential whole blood samples were collected for flow-cytometry phenotyping of immune cells, and for measuring systemic exposure to Ipi using ELISA

Results 40 patients were treated in the IT-arm and 21 in the IV-arm. The study met its primary endpoint with lower toxicity rate at 6 months in the IT-arm, with $30 \%[18.1 ; 45.4]$ vs $57.1 \%$ [36.5;75.5] of patients presenting $\geq$ grade 3 treatment related AEs, and no procedure-related $\geq$ grade 3 AEs in the IT-arm out of 162 IT injections performed (including deep seated lesions).ORR per RECIST 1.1 were observed in 50\% $[32.9 ; 67.1]$ in the IT-arm vs $65.0 \%[0.41 ; 0.85]$ in the IV-arm. In the IT-arm, $65.7 \%$ of the injected tumors showed a CR or PR.Serum Ipi concentrations were much lower in the IT arm (:10). At C2, patients in both arms had significant decreased circulating naïve Tregs independently from tumor responses. Presence of intratumoral CD25hi CD39hi activated Tregs that decreased significantly upon IT (but not IV) injection only in responders, was predictive of the overall tumor response in the IT-arm. Moreover, granzyme B concentrations in tumor secretome at baseline were higher in responders than non-responders in both arms.

Conclusions $0.3 \mathrm{mg} / \mathrm{kg}$ IT Ipi in combination with IV Nivo is not only safe but could reduce $\geq$ grade 3 toxicity of the ICB combination. The high response rate in injected lesions was associated with the reduction of local Treg -not observed with systemic Ipi- and prompts a use in the oligometastatic and neoadjuvant setting. Direct assessment of cytolytic and regulatory pathways on fresh biopsies represents a novel, simple and rapid strategy to predict treatment efficacy.

Acknowledgements Authors would like to thank the patients taking part to the Nivipit trial, and all the medical and paramedical staff that contributed to this trial.

Trial Registration NCT02857569EudraCT 2015-005429-37

Ethics Approval This study was approved by the national ethics committee (CPP, ANSM). Written informed consent was obtained from all patients.
Consent

N/A

http://dx.doi.org/10.1136/jitc-2021-SITC2021.277 